\title{
Determination of resolution in electron tomography
}

P. A. Penczek ${ }^{*}$ and J. Frank ${ }^{* *}$

* The University of Texas - Houston Medical School, Department of Biochemistry and Molecular Biology, 6431 Fannin, MSB 6.218, Houston, TX 77030, USA

${ }^{* *}$ HHMI, Health Research, Inc., Wadsworth Center, Albany, NY 12201, USA.

The determination of resolution in electron tomography requires a multifaceted approach. In order to decide whether a given reconstruction form EM projection data constitutes an acceptable approximation of the original (albeit unknown) structure it is necessary to consider a number of general issues: (i) the invertibility of the ray transform, particularly in the context of the slab geometry commonly used in electron tomography, (ii) the dependence of the distribution of projection angles on the data collection geometry, (iii) the influence of the limited angular range of the projection data, (iv) and the determination of the SSNR in the data.

The distribution of projection angles is derived based on the requirement for a possibly uniform coverage of the Fourier space by finite-thickness central sections corresponding to Fourier transforms of projections. For standard slab geometry of the sample in electron tomography, in single-axis tilt the angular step of projections has to decrease with the increased tilt angle. If the double-tilt data is collected, the same scheme has to be applied to the second tilt series, although low-tilt projections are redundant and should be omitted. The minimum tilt angle of the second tilt series is derived based on simple geometrical consideration. As a consequence, we show that for a maximum tilt angle of 70 degrees the number of second series projections that have to be collected is significantly reduced.

For the estimation of the SSNR in the reconstruction we introduce a novel approach based on the modified FSC analysis. We begin by noting that, given a set of noisy projections collected such that the Fourier space is sufficiently covered, it is impossible to state the resolution of the reconstruction unless the SSNR of the data is known. In the case of a 3-D reconstruction from 2-D projections (inversion of the ray transform) the object will necessarily have Fourier-space redundancies within the entire range of Fourier space. This is a simple consequence of the central section theorem: any non-trivial arrangement of central sections will share at least one common line and, as a result, will yield oversampled regions in Fourier space. By exploring these redundancies, and by using the standard FSC approach, it is possible: (i) to calculate the SSNR in certain regions of Fourier space, (ii) to calculate the SSNR in individual projections in the entire range of spatial frequencies, (iii) and, assuming isotropy of the data, to infer/deduce the resolution in non-redundant regions of Fourier space.

In the proposed approach the redundancies in Fourier space are used to calculate FSC coefficients within oversampled regions (Fig.1). This is done by comparing Fourier transforms of objects reconstructed from even- and odd-numbered projections, respectively. Because the degree of the oversampling in the region adjacent to tilt axis (or axes, for double tilt geometry) is high, it is possible to obtain a robust estimate of the FSC and, in consequence, the SSNR. Next, we have to assume that the SSNR in the data is isotropic and this allows us to infer what the SSNR in the undersampled regions of 
Fourier space is. Finally, given the known distribution of projections angles it becomes possible to calculate the distribution of the SSNR in the reconstructed 3-D object. We demonstrate the efficacy of the new method using a simple single-axis tilt simulated dataset. However, the method is quite general and applicable to all three data collection geometries used in electron tomography.

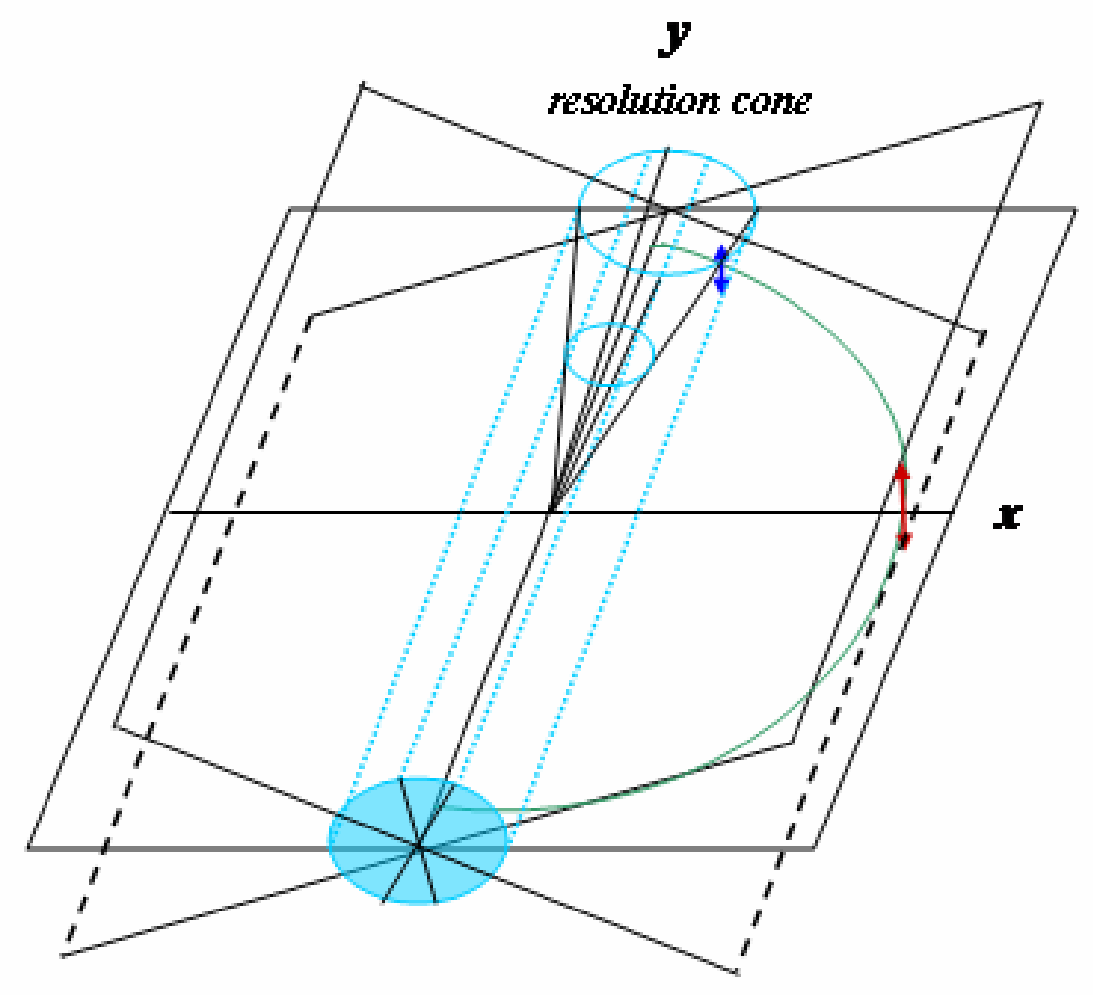

Figure 1. Estimation of SSNR of projection data and resolution of tomographic reconstruction. The half-circle - the region in 3-D Fourier space that has constant modulus of spatial frequency $|\mathrm{k}|$. Long vertical arrow - the larger gap in Fourier space in the direction perpendicular to the tilt axis $y$ at the spatial frequency $|\mathrm{k}|$. Short arrow - the smaller gap in Fourier space close to the position of the tilt axis $y$, also at the spatial frequency $|\mathrm{k}|$. The resolution is calculated using the modified FSC approach by carrying on the summation of Fourier coefficients located on circular cross sections of the resolution cone. For a small opening angle of the resolution cone, the oversampling of the Fourier space is approximately equal to the factor given by the number of projections.

Supported by the Grant NIH R01 GM 60635 (to P.A.P.) 